\title{
Tailored lead iodide growth for efficient flexible perovskite solar cells and thin-film tandem devices
}

\author{
Stefano Pisoni [1]', Romain Carron ${ }^{1}$, Thierry Moser ${ }^{1}$, Thomas Feurer ${ }^{1}$, Fan Fu ${ }^{1,2}$, Shiro Nishiwaki ${ }^{1}$, Ayodhya N. Tiwari ${ }^{1}$ and \\ Stephan Buecheler ${ }^{1}$
}

\begin{abstract}
Flexible perovskite solar cells (PSCs) hold great promise for the low-cost roll-to-roll production of lightweight singleand multijunction photovoltaic devices. Among the different deposition methods used for the perovskite absorber, the two-step hybrid vacuum-solution approach enables precise control over the thickness and morphology of $\mathrm{Pbl}_{2}$. However, efficient conversion to perovskite is limited by diffusion of the organic cations in the compact lead halide layer. Herein, a multistage absorber deposition is developed by thermal evaporation of $\mathrm{Pbl}_{2}$ and spin coating of $\mathrm{CH}_{3} \mathrm{NH}_{3} \mathrm{I}$ (MAl). The process relies on the different types of growth of vacuum-deposited $\mathrm{Pbl}_{2}$ onto amorphous and crystalline surfaces. This approach represents a way to effectively increase the absorber thickness while tackling the limited MAI diffusion in the compact $\mathrm{Pbl}_{2}$ film via a two-step deposition method. The efficiency of flexible PSCs is improved from 14.2 to $15.8 \%$ with multistage deposition. Furthermore, the use of an amorphous transparent conductive oxide (TCO), InZnO, enhances the mechanical resistance against bending with respect to conventional crystalline TCO-based flexible devices. Near-infrared transparent flexible PSCs are developed with an efficiency of $14.0 \%$ and average transmittance of $74 \%$ between 800 and $1000 \mathrm{~nm}$. Flexible perovskite/CIGS thin-film tandem devices are demonstrated with an efficiency of $19.6 \%$ measured in the four-terminal configuration.
\end{abstract}

\section{Introduction}

Solar cells developed on lightweight flexible substrates represent an interesting option for future energy generation applications. The possibility to produce flexible photovoltaic devices by high throughput roll-to-roll manufacturing opens up the way towards cost reduction compared to traditional sheet-to-sheet processes. Recently, hybrid organic-inorganic perovskites have emerged as a new generation of low-cost photovoltaic materials. The high absorption coefficient, high bandgap $(\sim 1.6 \mathrm{eV})$ and low Urbach energy make perovskites ideal

\footnotetext{
Correspondence: Stefano Pisoni (stefano.pisoni@empa.ch)

'Laboratory for Thin Films and Photovoltaics, Empa-Swiss Federal Laboratories for Materials Science and Technology, Ueberlandstrasse 129, 8600 Duebendorf, Switzerland

${ }^{2}$ now at Photovoltaics and Thin-Film Electronics Laboratory (PV-Lab), Ecole Polytechnique Fédérale de Lausanne (EPFL), Rue de la Maladière 71b, 2002 Neuchâtel, Switzerland
}

absorbers for single- and multijunction devices ${ }^{1,2}$. Among different perovskite-based multijunction technologies, all-thin-film perovskite/Cu(In,Ga)Se $\mathrm{Se}_{2}$ (CIGS) tandem devices have shown an outstanding performance improvement in the last three years, reaching efficiencies as high as $23.9 \%^{5}$. It has to be noted that the top and bottom cells in these tandem devices are grown onto rigid glass substrates. The demonstration of highly efficient flexible perovskite and CIGS solar cells ${ }^{6,7}$ has laid the foundations for flexible thin-film tandem devices ${ }^{8}$ with the promise of future roll-to-roll manufacturing of lightweight perovskite-based multijunction devices.

An interesting feature of perovskite solar cells (PSCs) is the wide versatility afforded by low-temperature deposition processes ${ }^{9-11}$. Among different deposition techniques, the hybrid vacuum-solution two-step method holds great potential for control of the $\mathrm{PbI}_{2}$ thickness and 
morphology to obtain compact and pinhole-free absorber layers ${ }^{12}$. Traditional solution-based processes, such as one- or two-step deposition, rely on the use of dimethylformamide (DMF) and dimethyl sulfoxide (DMSO) ${ }^{13}$. These solvents are not suitable with low-temperature deposited efficient organic-based electron transport layer (ETL) such as $\mathrm{C}_{60}{ }^{14}$. On the other hand, thermal evaporation of $\mathrm{PbI}_{2}$ followed by $\mathrm{CH}_{3} \mathrm{NH}_{3} \mathrm{I}$ (MAI) spin coating from isopropanol solution does not damage the very thin organic charge extraction layer. In a two-step deposition, the conversion to high-quality perovskite absorbers is dictated by the diffusion and intercalation of the organic cations into the inorganic $\mathrm{PbI}_{2}$ layered structure. Once the organic cation is deposited, the intercalation starts at the edge-air interface, where organic moieties immediately form hydrogen bonds with the inorganic sites. The thin surface layer of $\mathrm{CH}_{3} \mathrm{NH}_{3} \mathrm{PbI}_{3}$, which is suddenly formed, hinders further diffusion of MAI cations to the inner lead sites due to lack of Van der Waals gaps in the perovskite structure ${ }^{15,16}$. For this reason, a finely tailored $\mathrm{PbI}_{2}$ morphology is needed to facilitate MAI cation diffusion and play a fundamental role in the conversion to the perovskite absorber.

Different approaches have been reported to effectively engineer the $\mathrm{PbI}_{2}$ morphology, facilitating organic cation intercalation. Wu et al. ${ }^{17}$ showed the possibility to retard $\mathrm{PbI}_{2}$ compact-layer crystallization by coordination with DMSO molecules, improving the conversion to the perovskite phase. Tu et al. ${ }^{18}$ demonstrated solvent substitution to finely control the $\mathrm{PbI}_{2}$ nuclei growth and recrystallization process. Zhang et al. ${ }^{19}$ reported improved organic cation intercalation and conversion reaction by pre-expansion of the $\mathrm{PbI}_{2}$ layer using a mixed $\mathrm{PbI}_{2}$ :MAI precursor. Even if these approaches offer suitable solutions to significantly improve device efficiency, they cannot be applied when $\mathrm{PbI}_{2}$ is grown by vacuum deposition methods.

As we observed, in a hybrid two-step vacuum-solution process, the growth of thermally evaporated $\mathrm{PbI}_{2}$ depends on the substrate surface ${ }^{20}$. A compact morphology is observed once $\mathrm{PbI}_{2}$ is thermally evaporated onto amorphous surfaces due to a preferential growth parallel to the substrate as a result of minimization of combined surface and interface energy. On the other hand, a nanoplate-like morphology is formed onto crystalline substrates due to both the anisotropic nature of the substrate and interface energy $^{20}$.

Here we report a modified two-step hybrid vacuumsolution deposition method to effectively increase the absorber thickness. The morphology of $\mathrm{PbI}_{2}$ is tailored by a multistage deposition, where the first stage is characterized by a compact inorganic layer, and the second stage, by a nanoplate-like morphology. By careful screening of the organic cation concentration and nanoplate-like lead iodide thickness, we are able to demonstrate flexible PSC with a steady-state efficiency of $15.8 \%$ compared to a $14.2 \%$ efficient device prepared by a traditional two-step hybrid process.

For the transparent conductive oxide (TCO), we use sputtered InZnO (IZO), which displays high near-infrared transparency and an amorphous nature. The latter characteristic enables one to obtain highly flexible PSCs with respect to conventional flexible PSCs based on crystalline TCO. The devices retain 90 and $80 \%$ of the original efficiency after 1000 bending cycles at bending radii of 6 and $4 \mathrm{~mm}$, respectively. Furthermore, the high near-infrared transparency of the TCO makes it suitable for use in tandem applications. We are able to demonstrate a NIRtransparent flexible PSC with a steady state efficiency of $14.0 \%$ and average transmittance of $\sim 74 \%$ between 800 and $1000 \mathrm{~nm}$. By combining the flexible perovskite top cell with a flexible CIGS bottom cell, we demonstrate an efficiency of $19.6 \%$ measured in the four-terminal tandem configuration. Eventually, we present optical loss analysis to establish future steps towards realization of higherefficiency flexible perovskite/CIGS tandem devices.

\section{Materials and methods \\ Materials}

An InZnO target was bought from JX Nippon Mining \& Metals (99.9\%). Spiro-OMeTAD was bought from Merck. Polyethylenimine, $80 \%$ ethoxylated solution, 35-40 wt.\% in $\mathrm{H}_{2} \mathrm{O}$, 4-tertbutylpyridine (TBP) were bought from Sigma-Aldrich. $\mathrm{CH}_{3} \mathrm{NH}_{3} \mathrm{I}$ (powder, ITEM\# MS101000) and $\mathrm{PbI}_{2}$ (ultradry, 99.999\%, metals basis) were purchased from Dyesol (Australia) and Alfa Aesar, respectively. Fullerene carbon 60 powder $\left(\mathrm{C}_{60}\right)$ was bought from SES Research (purity $>99.5 \%$ ). All chemicals were used as received without any further treatment or purification.

\section{Photovoltaic device fabrication}

PSCs are grown onto a flexible foil, which is used as moisture barrier front sheet for encapsulation in flexible CIGS modules. Then, $5 \times 5 \mathrm{~cm}$ size flexible substrates are washed by hand followed by ultrasonic soap and water baths. The substrates are dried in vacuum for one week and cut into 4 quarters $(2.5 \times 2.5 \mathrm{~cm})$. Prior to further processing, $200 \mathrm{~nm}$ of compact InZnO (IZO) layer is deposited at room temperature by pulsed-DC sputtering from a ceramic $\operatorname{In}_{0.89} \mathrm{Zn}_{0.11} \mathrm{O}$ target, at $600 \mathrm{~W}$. The sheet resistance of the as-deposited film on glass is measured to be $18 \Omega \square$ by a four-probe method. Then, the IZO layer is treated for 10 min under UV light for all the samples. PEIE interlayer $(0.1 \% \mathrm{w} / \mathrm{w})$ in deionized (DI) water is deposited onto the IZO layer by spin coating (5000 r.p.m., $5000 \mathrm{rpm} \mathrm{s}^{-1}$ for $60 \mathrm{~s}$ ) followed by annealing at $100^{\circ} \mathrm{C}$ for $10 \mathrm{~min}$. Then, $\sim 5 \mathrm{~nm}$ of $\mathrm{C}_{60}$ is thermally evaporated in a $\mathrm{N}_{2}$ filled glovebox. The $\mathrm{PbI}_{2}$ film is thermally evaporated at a deposition 
pressure of $2-6 \times 10^{-6} \mathrm{~Pa}$. The deposition rate is controlled within 1.2-1.6 $\AA \mathrm{s}^{-1}$, monitored by a quartz crystal microbalance. The thickness of $\mathrm{PbI}_{2}$ is $140 \mathrm{~nm}$. After the $\mathrm{PbI}_{2}$ deposition, the samples are subsequently transferred into a $\mathrm{N}_{2}$-filled glove-box for further processing. The perovskite layer is formed by spin coating of $\mathrm{CH}_{3} \mathrm{NH}_{3} \mathrm{I}$ in 2propanol at a concentration of $55 \mathrm{mg} \mathrm{mL}^{-1}$. The solution is first spread to cover the whole substrate followed by waiting for $5 \mathrm{~s}$ before starting the rotation $(4000 \mathrm{rpm}$, $4000 \mathrm{rpm} \mathrm{s}-1$ for $40 \mathrm{~s}$ ). The as-prepared films are annealed at $100^{\circ} \mathrm{C}$ for $30 \mathrm{~min}$ on a hotplate inside the glovebox. In the case of multistage deposition, after perovskite annealing, a $\mathrm{PbI}_{2}$ film is thermally evaporated for the second time, following the same conditions used for the first stage. Different thicknesses have been investigated $(80,100,120$ and $140 \mathrm{~nm})$. Then, $\mathrm{CH}_{3} \mathrm{NH}_{3} \mathrm{I}$ in 2-propanol is spin-coated at different concentrations for every $\mathrm{PbI}_{2}$ thickness, as reported in the Results and discussion section. The as-prepared films are annealed at $100^{\circ} \mathrm{C}$ for $30 \mathrm{~min}$ on a hotplate inside the glovebox. After annealing, the samples are cooled down to room temperature, and $100 \mu \mathrm{L}$ of a Spiro-OMeTAD solution (78.2 mg 2,2',7,7'tetrakis-(N,N'-di- $p$-methoxyphenylamine)-9,9'-spirobifluorene (Spiro-OMeTAD), $33 \mu \mathrm{L}$ of a lithium-bis(trifluoromethanesulfonyl)imide (Li-TFSI) solution (170 mg Li-TFSI in $1 \mathrm{~mL}$ acetonitrile, Sigma-Aldrich)), and $8.2 \mu \mathrm{L}$ of 4-tertbutylpyridine (TBP) all dissolved in $1 \mathrm{~mL}$ of chlorobenzene (Sigma-Aldrich) are spin coated on top of the perovskite at $2500 \mathrm{rpm}$ and $2500 \mathrm{rpm} \mathrm{s}-1$ for $45 \mathrm{~s}$. The devices are finished by evaporating $50 \mathrm{~nm}$ of Au through a metal mask under high vacuum $\left(<3 \times 10^{-4} \mathrm{~Pa}\right)$. The solar cell area is equal to $0.15 \mathrm{~cm}^{2}$.

For NIR-transparent flexible PSCs, an 20-nm-thick $\mathrm{MoO}_{\mathrm{x}}$ layer is deposited on top of the Spiro-OMeTAD via thermal evaporation $\left(<3 \times 10^{-4} \mathrm{~Pa}\right)$, which is then covered by $\sim 200 \mathrm{~nm}$ of IZO as the transparent electrode ( $200 \mathrm{~W})$. $\mathrm{Ni} / \mathrm{Al}$ grids with a $50 \mathrm{~nm} / 4000 \mathrm{~nm}$ thickness are deposited by e-beam evaporation. The solar cell area is defined by mechanical scribing.

\section{Characterization}

The current density-voltage characteristics of PSCs are measured under standard simulated AM1.5 G illumination using a Keithley 2400 source meter. The illumination intensity is calibrated to $1000 \mathrm{~W} \mathrm{~m}^{-2}$ using a certified single-crystalline silicon solar cell. The $J-V$ measurement is performed in both the forward (from -0.1 to $1.4 \mathrm{~V}$ ) and backward (from 1.4 to $-0.1 \mathrm{~V}$ ) direction separately without any pretreatment (e.g., light soaking, holding at forward bias for a certain time, etc.). The scan rate and delay time are $0.3 \mathrm{~V} \mathrm{~s}^{-1}$ and $10 \mathrm{~ms}$, respectively. The external quantum efficiency is measured with a lock-in amplifier. The probing beam is generated by a chopped white source $(900 \mathrm{~W}$, halogen lamp, $260 \mathrm{~Hz})$ and a dual-grating monochromator. The beam size is adjusted to ensure that the illumination area is fully inside the cell area. A certified single-crystalline silicon solar cell is used as the reference cell. White light bias is applied during the measurement with $\sim 0.1$ sun intensity. The steady-state efficiency as a function of time is recorded using a maximum power point tracker, which adjusts the applied voltage to reach the maximum power point (perturb and observe algorithm). The starting voltage is set to be $0.1 \mathrm{~V}$. The tandem device in the four-terminal configuration is characterized as reported elsewhere ${ }^{12}$.

The bending tests are carried out using metallic cylinders to define the curvature radius. Cylinders with a diameter of $12 \mathrm{~mm}$ and $8 \mathrm{~mm}$ are used for bending with radii of $6 \mathrm{~mm}$ and $4 \mathrm{~mm}$, respectively. The devices are bent repeatedly on these cylinders.

X-ray diffraction patterns were obtained in the BraggBrentano geometry by using a X'Pert PRO $\theta-2 \theta$ scan $(\mathrm{Cu}-$ $K_{\alpha 1}$ radiation, $\lambda=1.5406 \AA$ ) from 10 to $60^{\circ}(2 \theta)$ with a step interval of $0.0167^{\circ}$.

The SEM images of the samples were investigated with a Hitachi using a voltage and current of $5 \mathrm{kV}$ and $10 \mathrm{~mA}$, respectively. A thin layer of $\mathrm{Pt}(1 \mathrm{~nm})$ was coated on top of the sample to avoid the charging effect.

The morphology images were acquired using an AFM Bruker ICON operated in PeakForce QNM mode (based on PeakForce Tapping technology).

Reflectance and transmittance were measured using a UV-3600 Shimadzu UV-VIS-NIR spectrophotometer equipped with an integrating sphere. Ellipsometry measurements were carried out with a Woollam M2000 VNIR instrument in a wavelength range of $370-1670 \mathrm{~nm}$ at incidence angles of $50^{\circ}, 60^{\circ}$ and $70^{\circ}$. The refractive indices of the different layers were determined by processing of reflectance, transmittance and ellipsometry data using the RefFit software ${ }^{21}$. The dielectric functions were constructed as the sums of Lorentz and Tauc-Lorentz oscillators, ensuring consistency with the Kramers-Kroning relations. The optical propagation in the solar cell was simulated using the transfer matrix method (TMM) implemented in the tmm python package ${ }^{22}$.

\section{Results and discussion}

The flexible substrate used in this work is commonly applied as an encapsulation front sheet for flexible CIGS solar modules, and it displays ultralow optical absorptance and water vapor transmission rate, as reported previously ${ }^{8}$. The use of this particular substrate opens up the way to the direct integration of a flexible perovskite top cell in a traditional flexible CIGS module manufacturing line. Supplementary Figure S1a shows the optical properties of the flexible substrate coated with an indium zinc oxide (IZO) transparent conductive oxide (TCO) layer. The average absorptance of the flexible substrate/TCO 
layer stack is $\sim 5 \%$ between 400 and $1000 \mathrm{~nm}$. The IZO deposited by rf-magnetron sputtering shows a carrier mobility of $54.8 \mathrm{~cm}^{2} \mathrm{~V}^{-1} \mathrm{~s}^{-1}$, as determined by Hall measurements. As shown in Supplementary Figure S1b, from XRD analysis, the TCO layer is found to be amorphous, in accordance with other studies ${ }^{23}$.

A combination of spin-coated poly(ethylenimine) ethoxilated (PEIE) and thermally evaporated $\mathrm{C}_{60}$ is used to enhance charge extraction and collection from the absorber to the anode ${ }^{24}$.

The perovskite absorber is deposited using a two-step hybrid vacuum-solution deposition process ${ }^{12} . \mathrm{PbI}_{2}$ is thermally evaporated and converted to $\mathrm{CH}_{3} \mathrm{NH}_{3} \mathrm{PbI}_{3}$ by spin coating $\mathrm{CH}_{3} \mathrm{NH}_{3} \mathrm{I}$ (MAI) in 2-propanol solution. Supplementary Figure S2a shows a cross-sectional SEM image of the flexible PSC using a two-step hybrid process. As shown in Supplementary Figure S2b, we are able to demonstrate a flexible PSC, which shows an open-circuit voltage $\left(V_{\mathrm{oc}}\right)$ of $1.07 \mathrm{~V}$, a short-circuit current $\left(J_{\mathrm{sc}}\right)$ of $18.5 \mathrm{~mA} \mathrm{~cm}^{-2}$ and a fill factor (FF) of $72.9 \%$ under forward measurement while also showing a $V_{\text {oc }}$ of $1.05 \mathrm{~V}, J_{\mathrm{sc}}$ of $18.3 \mathrm{~mA} \mathrm{~cm}^{-2}$ and $\mathrm{FF}$ of $72.1 \%$ under backward measurement. The cell is tested at the maximum power point (MPP) under continuous illumination to evaluate the efficiency under operating conditions. The device shows a power conversion efficiency of $14.2 \%$ (Supplementary Figure S2c). Supplementary Figure S2d shows the external quantum efficiency (EQE) of the device, with the integrated $J_{\mathrm{sc}}$ value for AM1.5 G determined to be $18.5 \mathrm{~mA}$ $\mathrm{cm}^{-2}$.

Our samples contain residual $\mathrm{PbI}_{2}$ (Supplementary Figure S2e, $\sim 12.7^{\circ}$ ), which has been confirmed to be beneficial for suppressing non radiative recombination ${ }^{25}$.

In comparison to state-of-the-art of flexible PSCs ${ }^{6}$, the main bottleneck in our device is represented by the low $J_{\mathrm{sc}}$ values. One of the main causes for the low current yield is related to the limited absorption of the thin perovskite layer $(<300 \mathrm{~nm})$ deposited in the two-step hybrid process (Supplementary Figure S2a). A straightforward solution would be to simply increase the perovskite absorber thickness by deposition of a thicker $\mathrm{PbI}_{2}$ layer.

As reported by other studies, for a two-step deposition method, increasing the thickness of the perovskite absorber does not result in direct efficiency improvement ${ }^{26-28}$. Having a thick and compact $\mathrm{PbI}_{2}$ layer hinders efficient diffusion and intercalation of organic cations. By increasing the thickness of the lead iodide, higher charge transport resistance and stronger charge recombination have been observed ${ }^{27}$.

We increased the $\mathrm{PbI}_{2}$ thickness to $200 \mathrm{~nm}$ to eventually obtain a thicker perovskite layer. Different MAI concentrations were investigated: 70,75 and $80 \mathrm{mg} \mathrm{mL}^{-1}$ (Supplementary Figure S3). Our results show that an increase in the inorganic layer thickness does not result in efficiency improvements with respect to optimum thinner absorber thicknesses (Supplementary Figure S2). As the organic precursor concentration is increased, $J_{\mathrm{sc}}$ increases, saturating at higher concentrations, in contrast with an observed decrease in FF values. A too low MAI concentration results in limited conversion to the perovskite phase. On the other hand, a certain amount of unreacted lead iodide has been confirmed to be beneficial for device performance ${ }^{25,29}$. For this reason, a too large amount of MAI must be avoided, considering the detrimental effects observed due to an excess of uncoordinated $\mathrm{MA}^{+}$ions ${ }^{30}$.

To effectively increase the absorber thickness, and consequently the device efficiency, we believe that the MAI intercalation needs to be facilitated by appropriate tailoring of the $\mathrm{PbI}_{2}$ morphology. Figure 1 shows a schematic for the proposed multistage deposition protocol. Thermally evaporated $\mathrm{PbI}_{2}$ on top of amorphous $\mathrm{C}_{60}$ grows in a compact morphology with a root mean square (RMS) roughness of $\sim 9 \mathrm{~nm}$ as measured by AFM. The isotropic nature of the substrate and interface surface energy favors a preferential growth parallel to the substrate $^{20}$. Once the organic precursor is deposited and the film is annealed, a compact perovskite layer forms with a surface RMS roughness of $\sim 22 \mathrm{~nm}$. The proposed modified process relies on the vacuum deposition of $\mathrm{PbI}_{2}$ on top of the perovskite. The absorber displays a crystalline surface. The anisotropy of both the substrate and interface surface energy dictates the formation of a noncompact nanoplate-like $\mathrm{PbI}_{2}$ morphology ${ }^{20}$. This open framework will facilitate efficient organo-halide diffusion and intercalation in the following spin coating step. The nanoplatelike morphology is verified by SEM, with the RMS roughness of the inorganic layer deposited on top of the perovskite found to be $\sim 28 \mathrm{~nm}$. Eventually, the organic precursor is deposited by spin coating. The absorber layer obtained via the multistage process shows a compact morphology without a clear separation between the two deposition stages. The RMS roughness of the final perovskite absorber is found to be equal to $\sim 37 \mathrm{~nm}$.

As there is an optimum proportion between the compact $\mathrm{PbI}_{2}$ layer thickness and organic cation concentration, an optimum ratio has to be defined for the nanoplate-like $\mathrm{PbI}_{2}$ layer thickness as well. It is necessary to provide an appropriate concentration of organic cations for a given inorganic layer thickness. This concentration must be sufficient to break the weak Van der Waals forces between the layers of $\mathrm{PbI}_{2}$ and convert them into $\mathrm{PbI}_{6}$ octahedral networks. Meanwhile, the concentration should not be too high to avoid steric hindrance and an excess of unreacted organic moieties.

Different combinations of second-stage nanoplate-like $\mathrm{PbI}_{2}$ layer thicknesses and MAI concentrations were investigated and compared. Supplementary Figure S4a shows multistage deposition with $80 \mathrm{~nm}$ of $\mathrm{PbI}_{2}$ as the 


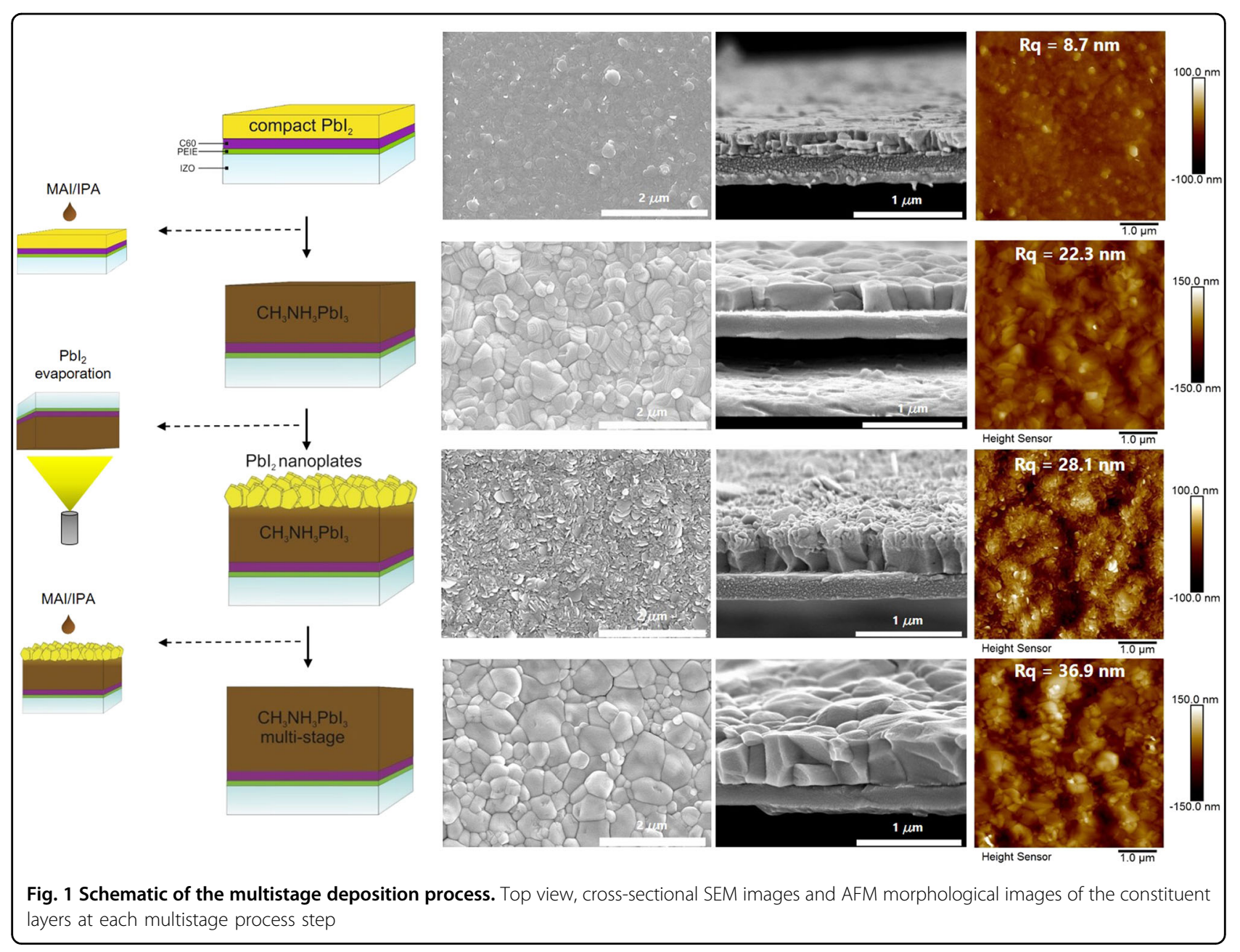

second step. The MAI concentration was changed from 20 to $30 \mathrm{mg} \mathrm{mL}^{-1}$, with an efficiency of $15.3 \%$ (under MPP tracking) obtained for a concentration of $25 \mathrm{mg} \mathrm{mL}^{-1}$. Supplementary Figure S4b presents the device performance with $100 \mathrm{~nm}$ of $\mathrm{PbI}_{2}$ and MAI concentrations that vary from 30 to $40 \mathrm{mg} \mathrm{mL}^{-1}: 35 \mathrm{mg} \mathrm{mL}^{-1}$ of MAI leads to an efficiency of $15.4 \%$. Supplementary Figure S4c shows deposition with $140 \mathrm{~nm}$ of $\mathrm{PbI}_{2}$ and MAI concentrations varying from 45 to $55 \mathrm{mg} \mathrm{mL}^{-1}$, with the best efficiency of $14.7 \%$ reached at $50 \mathrm{mg} \mathrm{mL}^{-1}$. The very thick absorber results in poorer device performance due to limited carrier diffusion length in the pure $\mathrm{MAPI}_{3}$ perovskite ${ }^{31}$.

The optimum proportion is achieved with a 120 -nm-thick of nanoplate-like $\mathrm{PbI}_{2}$ layer (Fig. 2a) and with $45 \mathrm{mg} \mathrm{mL}^{-1}$ of MAI in the second stage (Fig. 2b). We are able to demonstrate a flexible PSC with a steady-state efficiency of $15.8 \%$ (Fig. 2c). The device shows a $V_{\text {oc }}$ of $1.07 \mathrm{~V}, J_{\mathrm{sc}}$ of $19.7 \mathrm{~mA} \mathrm{~cm}^{-2}$ and an FF of $74.0 \%$ when measured in the forward direction and a $V_{\text {oc }}$ of $1.06 \mathrm{~V}, J_{\mathrm{sc}}$ of $19.6 \mathrm{~mA} \mathrm{~cm}^{-2}$ and an $\mathrm{FF}$ of $73.4 \%$ when measured in the backward direction. As can be observed from the EQE measurements (Fig. 2d), a significant improvement with respect to the two-step hybrid process is achieved at long wavelengths, indicating stronger absorption in the perovskite layer. The final device displays a compact absorber layer that contains residual $\mathrm{PbI}_{2}$ (Fig. 2e, f).

From an application point of view, mechanical resistance against bending is pivotal to ensure working flexible photovoltaic devices. The bottleneck of flexibility dwells in the brittle TCO, which is usually crystalline ITO $^{32}$. It is observed that when crystalline ITO is used as the TCO, flexible PSC performance decreases well below $60 \%$ of the initial value after 1000 cycles at a bending radius of $4 \mathrm{~mm}^{33}$. Different strategies have been proposed to overcome this problem. Among them, amorphous TCO films deposited at low temperature have been used to enhance flexibility in organic photovoltaics. It has been shown that amorphous films have a robust structure against external dynamic stresses compared with crystalline films $s^{34,35}$.

In our flexible perovskite device structure, we employ $\sim 200 \mathrm{~nm}$ of amorphous IZO as the TCO. To demonstrate mechanical stability against bending, we tested cells at bending radii of $6 \mathrm{~mm}$ and $4 \mathrm{~mm}$ for 1000 bending cycles. 
(a)

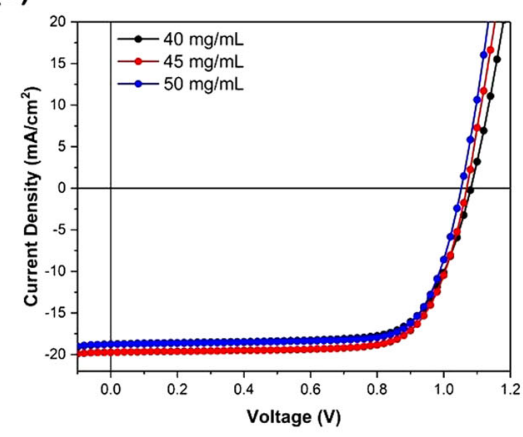

(d)

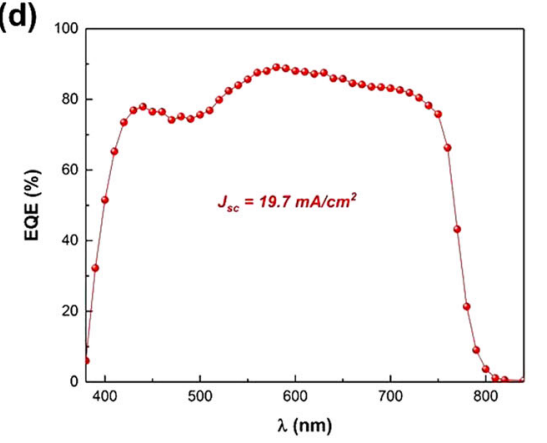

(b)

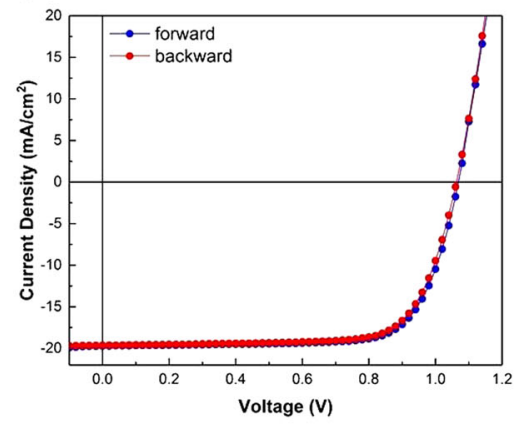

(e)

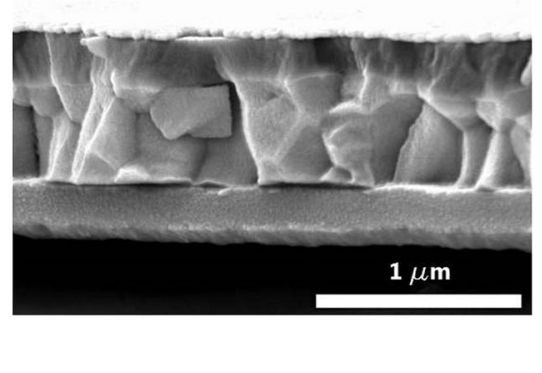

(c)

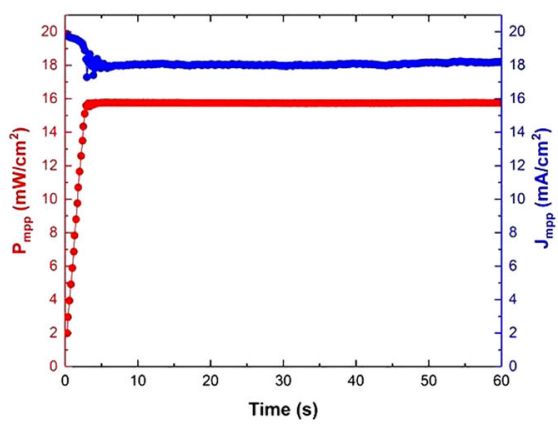

(f)

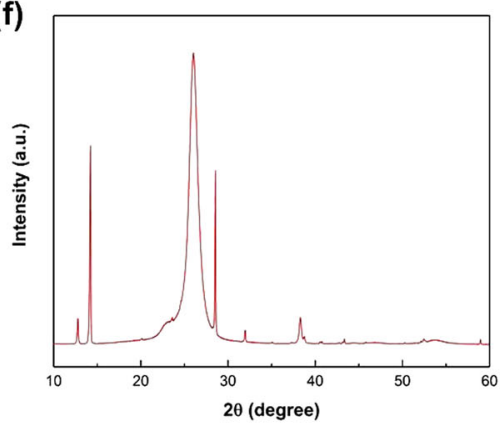

Fig. 2 Flexible perovskite solar cell with multistage absorber deposition. a Comparison of the $J-V$ characteristics obtained for devices with $120 \mathrm{~nm}$ of $\mathrm{Pbl}_{2}$ and varying MAl concentration $\left(40,45\right.$, and $50 \mathrm{mg} \mathrm{mL}^{-1}$ ) in the second step of multistage deposition. $\mathbf{b} \mathrm{J}-V$ characteristics measured under a forward (blue line) and backward (red line) scan for the best device prepared using multistage deposition. c Power conversion efficiency (red line) and current density output (blue line) measured by MPP tracking under one sun continuous illumination. $\mathbf{d}$ EQE spectrum for the flexible perovskite solar cell prepared using multistage deposition. e SEM cross-section of the flexible device, scale bar $1 \mu$ m. $\mathbf{f}$ XRD graph of the flexible perovskite device

Supplementary Figure S5a shows stability against a bending radius of $6 \mathrm{~mm}$. The device displays an efficiency at 1000 bending cycles that is $\sim 90 \%$ of the initial power conversion efficiency. Stability test against a bending radius of $4 \mathrm{~mm}$ is presented in Supplementary Figure S5b. The device retains more than $80 \%$ of the original efficiency at 1000 bending cycles. These bending performances exceed those consistently obtained in the literature using crystalline ITO as the $\mathrm{TCO}^{32,33}$. The achievable robustness to bending is controlled by a complex interplay among defects, residual film stresses, cohesive properties, and so forth. Furthermore, the suppression of crystallization is required to obtain a stressfree or reduced-stress film ${ }^{34,36}$.

For application in four-terminal tandem devices, the top cell requires a near-infrared (NIR) transparent back electrode to guarantee that low-energy photons can reach the bottom cell. For the transparent electrode, a combination of $\mathrm{MoO}_{x}$ and IZO was used. Figure 3a shows the SEM cross-section of the NIR-transparent flexible PSC using multistage deposition. The cell displays an average transmittance of $\sim 74 \%$ between 800 and $1000 \mathrm{~nm}$ (Fig. 3b). The device shows a $V_{\mathrm{oc}}$ of $1.06 \mathrm{~V}, J_{\mathrm{sc}}$ of $18.7 \mathrm{~mA} \mathrm{~cm}^{-2}$ and an FF of $69.0 \%$ when measured in forward direction and a
$V_{\text {oc }}$ of $1.04 \mathrm{~V}, J_{\mathrm{sc}}$ of $18.6 \mathrm{~mA} \mathrm{~cm}^{-2}$ and an $\mathrm{FF}$ of $69.4 \%$ when measured in the backward direction (Fig. 3c). The NIR-transparent solar cell reaches an efficiency of $14.0 \%$ at the MPP under one-sun illumination (Fig. 3d). The integrated $J_{\text {sc }}$ value determined from EQE (Fig. 3e) is $18.7 \mathrm{~mA} \mathrm{~cm}^{-2}$. We demonstrate a perovskite/CIGS tandem device in the four-terminal configuration, where the top and bottom cells are grown on flexible substrates. Figure $4 \mathrm{a}, \mathrm{b}$ displays the $J-V$ curves and $\mathrm{EQE}$ spectra for the top PSC, CIGS standalone cell and CIGS bottom cell. Table 1 summarizes the photovoltaic parameters of the corresponding devices. The flexible perovskite/CIGS tandem device measured in four-terminal configuration shows an efficiency of $19.6 \%$, starting from an $18.9 \%$ flexible CIGS solar cell.

It is clear that increasing the absorption of the top cell results in a lower bottom cell current yield, which could limit the final tandem device performance. For this reason, we decided to compare the multistage perovskite deposition with two-step hybrid deposition for top cell application. The $J-V$ characteristics of the latter NIR-transparent flexible PSC show a $V_{\mathrm{oc}}$ of $1.10 \mathrm{~V}, J_{\mathrm{sc}}$ of $17.5 \mathrm{~mA} \mathrm{~cm}^{-2}$ and FF of $68.1 \%$ under the forward scan and a $V_{\mathrm{oc}}$ of $1.08 \mathrm{~V}, J_{\mathrm{sc}}$ of $17.4 \mathrm{~mA} \mathrm{~cm}^{-2}$ and $\mathrm{FF}$ of $68.9 \%$ when measured under the 

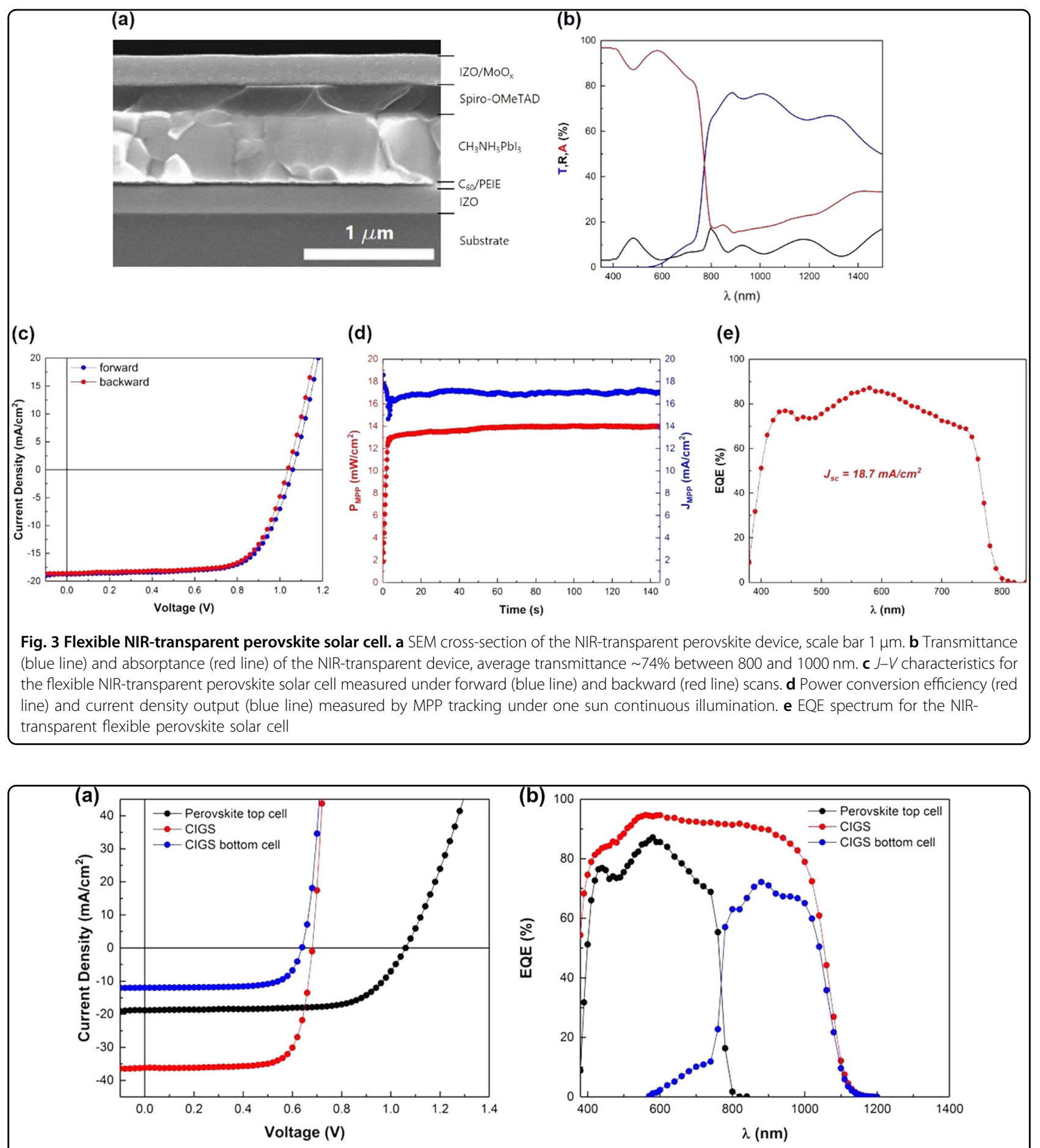

Fig. 4 Flexible perovskite/CIGS tandem cell measured in the four-terminal configuration. a $J-V$ characteristics of the flexible CIGS standalone cell (red curve), flexible CIGS bottom cell (blue curve) and flexible perovskite top cell (dark curve). b EQE spectra for the corresponding devices

backward scan (Supplementary Figure S6a). The device displays a steady-state efficiency of $13.1 \%$ (Supplementary Figure S6b). The NIR-transparent device, developed by the two-step hybrid process, shows a lower current yield with respect to multistage deposition. The reason for this is due to the lower absorption in the long wavelength region of the EQE (Supplementary Figure S6c). Combining the perovskite top cell with the flexible CIGS bottom cell (Supplementary Table S1), an efficiency of $19.2 \%$ is achieved (Supplementary Figure S6d, e). Although the bottom cell 
Table $1 \mathrm{~J}-V$ parameters for each constituent solar cell in the tandem device

\begin{tabular}{|c|c|c|c|c|c|c|}
\hline Solar cell & $V_{\text {oc }}(\mathrm{V})$ & $J_{\mathrm{scv}}\left(\mathrm{mA} \mathrm{cm} \mathrm{cm}^{-2}\right)$ & FF (\%) & $\eta(\%)$ & $\eta_{\mathrm{MPP}}(\%)$ & Area $\left(\mathrm{cm}^{2}\right)$ \\
\hline Flexible CIGS & 0.69 & 36.3 & 75.4 & 18.9 & 18.9 & 0.213 \\
\hline Flexible CIGS bottom cell & 0.65 & 12.0 & 71.6 & 5.6 & 5.6 & 0.213 \\
\hline Flexible perovskite top cell & 1.06 & 18.7 & 68.9 & 13.7 & 14.0 & 0.27 \\
\hline Flexible perovskite/CIGS four-terminal tandem configuration & & & & & 19.6 & \\
\hline
\end{tabular}

efficiency is greater (due to the higher absorption in the CIGS), the tandem device with a multistage top cell still outperforms the device developed using the two-step hybrid process due to the higher performance of the top cell.

To assess potential pathways to further improve flexible perovskite/CIGS tandem solar cells, we carried out optical analysis of the NIR-transparent perovskite top cell structure as an example of a low-temperature $n-i-p$ PSC that can be deposited on flexible substrates (Fig. 5a). The transfer matrix method (TMM) was used to simulate the reflectance, transmittance of the multilayer structure and the absorption spectra of the different layers. The layers were considered optically flat (no interface roughness was taken into account). Complex refractive indices were either characterized in house (IZO front and rear TCO, PEIE, $\mathrm{C}_{60}$ and $\mathrm{MoO}_{\mathrm{x}}$ ) or taken from the literature $\left(\mathrm{MAPbI}_{3}\right.$ and Spiro-OMeTAD) ${ }^{37}$. Supplementary Figure S7 presents experimental data and best fits to the transmittance, reflectance and ellipsometry spectra of the layers used for the determination of the refractive indices. Supplementary Figure S8 displays experimental and TMM-based simulations of the reflectance, transmittance and absorptance of the NIR-transparent perovskite top cell in the visible range, with the layer thicknesses reported in Supplementary Table S2. The discrepancies between experimental and simulated reflectance (and absorptance) are related to light scattering and to the different substrates considered in the simulations with respect to the actual substrate (notably, including the encapsulation front sheet with specific oxide coatings). The optical losses in each of the layers can be evaluated in terms of the corresponding losses in the short-circuit current (Table S2) using AM1.5 G irradiance. As shown in Fig. 5b, the presence of the IZO front TCO and the ETL (PEIE interlayer and $\mathrm{C}_{60}$ ) leads to the absorption of light that cannot reach the perovskite absorber. The corresponding $J_{\mathrm{sc}}$ losses in the front TCO and ETL amount to 1.0 and $0.2 \mathrm{~mA} \mathrm{~cm}^{-2}$, respectively. This loss due to parasitic absorption can be mitigated by using an improved front TCO with comparatively lower absorption (our IZO presents nonnegligible absorption in the visible) and by the use of a more transparent low-temperature ETL (as inorganic ones). Reflection losses can be observed especially between $450-500 \mathrm{~nm}$, which can be reduced by an appropriate anti-reflection (AR) coating, such as a transparent layer with a refractive index intermediate between that of air and the substrate and/or the substrate and the front TCO. At longer wavelengths, reflectance can be reduced by using an AR coating at the rear TCO/air interface. For $\lambda>$ $600 \mathrm{~nm}$, the NIR-transparent solar cell presents transmission losses due to the absence of an internal back reflector typical of opaque devices (metal back contact). In this region $(600-800 \mathrm{~nm})$, the effect on the transmittance of the layers deposited after $\mathrm{MAPbI}_{3}$ is negligible, such that the photons lost in this wavelength range are efficiently absorbed by the bottom cell, as shown in Fig. $4 \mathrm{~b}$. Lastly, hints to the collection and recombination losses are obtained by combining the experimental and simulation data, as described by Armin et al. ${ }^{38}$. In the case of our NIR-transparent cell, the sum of the experimental EQE with the predicted parasitic absorption in the front layers does not quite reach the level of the experimental absorptance of the device, as shown in Fig. 5b. This difference is seemingly independent from the excitation wavelength and is tentatively ascribed to collection losses and/or to recombination. We believe that these losses can be mitigated by proper interface engineering and by processing of higher-quality perovskite absorbers.

\section{Conclusion}

We have demonstrated a modified hybrid vacuumsolution deposition method to tailor $\mathrm{PbI}_{2}$ growth for effectively increasing the perovskite thickness. Through multistage deposition, an improvement in performance for flexible PSCs, from a steady-state efficiency of 14.2 to $15.8 \%$, was shown. Using an amorphous TCO (IZO), we were able to demonstrate superior bending stability with respect to the traditional crystalline TCO used for flexible PSCs. Flexible devices retained 90 and $80 \%$ of the initial efficiency after 1000 bending cycles at bending radii of 6 and $4 \mathrm{~mm}$, respectively. We have developed a flexible NIR-transparent PSC with a steady-state efficiency of $14.0 \%$. The devices were grown onto a flexible foil, which is used as an encapsulation front sheet for CIGS solar modules. By combining the flexible perovskite top cell with a flexible CIGS bottom cell, a flexible perovskite/ CIGS tandem cell, with an efficiency of $19.6 \%$ measured in the four-terminal configuration, was demonstrated. 

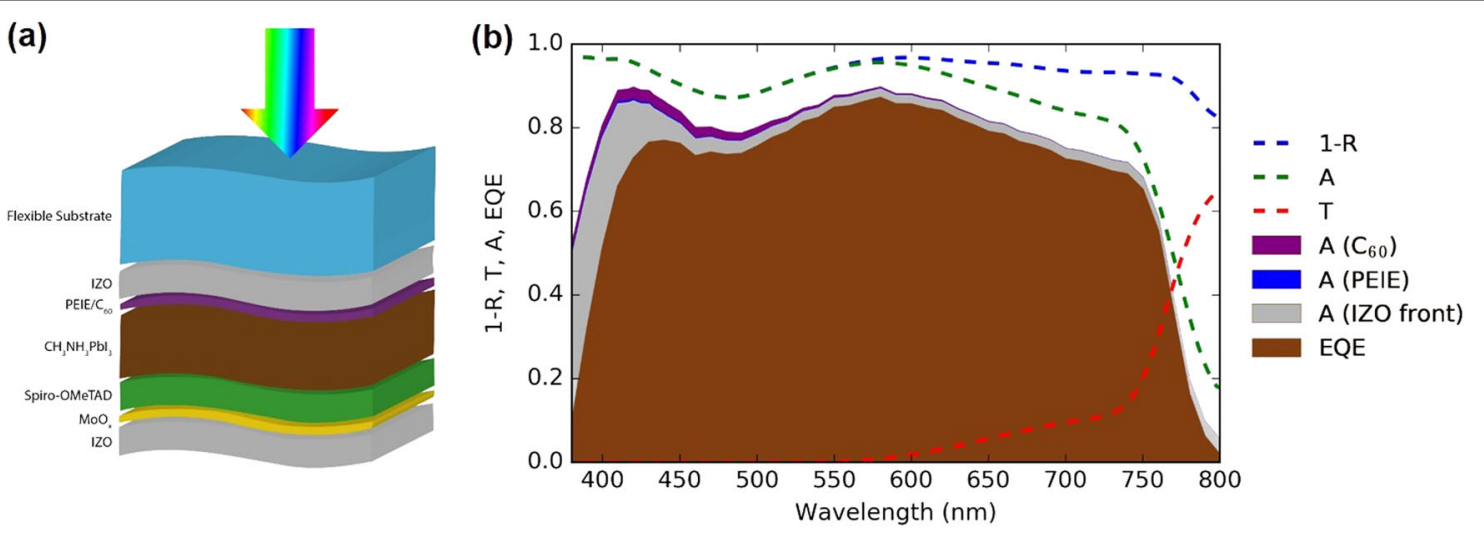

Fig. 5 Optical analysis of the NIR-transparent perovskite top cell structure. a Schematic of the NIR-transparent flexible perovskite top cell in the superstrate configuration. b Optical analysis of the NIR-transparent PSC. The experimental reflectance, transmittance, and absorptance are represented as dashed lines. The experimental EQE and the parasitic absorption in the top layers obtained from simulations are illustrated with filled curves. The simulated absorption in the IZO, PEIE and $\mathrm{C}_{60}$ front layers (Supplementary Figure S7) are stacked on top of the experimental EQE

Through analyses of the optical losses and TMM-based simulations, we identified the next steps towards realizing higher efficiency NIR-transparent flexible PSCs.

In this work, we described a method to engineer the $\mathrm{PbI}_{2}$ layer morphology using vacuum deposition. The multistage deposition protocol widens the potential for use of the hybrid vacuum-solution process towards the realization of highly efficient devices by precise morphological tuning.

\section{Acknowledgements}

This work was financial funded by the Swiss National Science Foundation (SNF)-NRP70, PV2050 (project NO:: 407040 153976 and 407040 153916), SNFNanoTera and Swiss Federal Office of Energy (SYNERGY: 20NA21_150950), NanoTera (project Synergy Gateway) and FP7 APPOLO project (609355). We acknowledge the access to the Scanning Probe Microscopy User Lab at Empa for the (AFM) measurements. A special thank you goes to Marc Padrutt, a visiting student in our group.

\section{Conflict of interest}

The authors declare that they have no conflict of interest.

\section{Publisher's note}

Springer Nature remains neutral with regard to jurisdictional claims in published maps and institutional affiliations.

Supplementary information is available for this paper at https://doi.org/ 10.1038/s41427-018-0099-1.

Received: 14 July 2018 Revised: 11 September 2018 Accepted: 1 October 2018.

Published online: 16 November 2018

\section{References}

1. Green, M. A., Ho-Baillie, A. \& Snaith, H. J. The emergence of perovskite solar cells. Nat. Photonics 8, 506 (2014).

2. De Wolf, S. et al. Organometallic halide perovskites: sharp optical absorption edge and its relation to photovoltaic performance. J. Phys. Chem. Lett. 5, 1035-1039 (2014).
3. Werner, J., Niesen, B. \& Ballif, C. Perovskite/silicon tandem solar cells: marriage of convenience or true love story? - an overview. Adv. Mater. Interfaces $\mathbf{5}$, 1700731 (2018).

4. Eperon, G. E., Hörantner, M. T. \& Snaith, H. J. Metal halide perovskite tandem and multiple-junction photovoltaics. Nat. Rev. Chem. 1, 0095 (2017).

5. Shen, H. et al. Mechanically-stacked perovskite/CIGS tandem solar cells with efficiency of 23.9\% and reduced oxygen sensitivity. Energy Environ. Sci. 11, 394-406 (2018).

6. Bi, C., Chen, B., Wei, H., DeLuca, S. \& Huang, J. Efficient flexible solar cell based on composition-tailored hybrid perovskite. Adv. Mater. 29, 1605900 (2016).

7. Chirilă, A. et al. Potassium-induced surface modification of $\mathrm{Cu}(\mathrm{In}, \mathrm{Ga}) \mathrm{Se}_{2}$ thin films for high-efficiency solar cells. Nat. Mater. 12, 1107-1111 (2013).

8. Pisoni, S. et al. Flexible NIR-transparent perovskite solar cells for all-thin-film tandem photovoltaic devices. J. Mater. Chem. A 5, 13639-13647 (2017).

9. Jung, H. S. \& Park, N-G. Perovskite solar cells: from materials to devices. Small 11, 10-25 (2015).

10. Momblona, C. et al. Efficient vacuum deposited $\mathrm{p}-\mathrm{i}-\mathrm{n}$ and n-i-p perovskite solar cells employing doped charge transport layers. Energy Environ. Sci. 9, 3456-3463 (2016).

11. Ávila, J., Momblona, C., Boix, P. P., Sessolo, M. \& Bolink, H. J. Vapor-deposited perovskites: the route to high-performance solar cell production? Joule $\mathbf{1}$, 431-442 (2017).

12. Fu, F. et al. Low-temperature-processed efficient semi-transparent planar perovskite solar cells for bifacial and tandem applications. Nat. Commun. $\mathbf{6}$, 8932 (2015)

13. Yang, W. S. et al. lodide management in formamidinium-lead-halide-based perovskite layers for efficient solar cells. Science 356, 1376-1379 (2017).

14. Wojciechowski, K. et al. C60 as an efficient n-type compact layer in perovskite solar cells. J. Phys. Chem. Lett. 6, 2399-2405 (2015).

15. Ahmad, S., Kanaujia, P. K., Niu, W., Baumberg, J. J. \& Vijaya Prakash, G. In situ intercalation dynamics in inorganic-organic layered perovskite thin Films. ACS Appl. Mater. Interfaces 6, 10238-10247 (2014).

16. Mitzi, D. B. in Progression in Inorganic Chemistry (ed Mitzi, D. B.) 1-121 WileyBlackwell, 2007), Canada.

17. $\mathrm{Wu}, \mathrm{Y}$. et al. Retarding the crystallization of $\mathrm{Pbl} 2$ for highly reproducible planar-structured perovskite solar cells via sequential deposition. Energy Environ. Sci. 7, 2934-2938 (2014).

18. $\mathrm{Tu}, \mathrm{Y}$. et al. Solvent engineering for forming stonehenge-like $\mathrm{Pbl} 2$ nanostructures towards efficient perovskite solar cells. J. Mater. Chem. A 5 4376-4383 (2017).

19. Zhang, T., Yang, M., Zhao, Y. \& Zhu, K. Controllable sequential deposition of planar $\mathrm{CH} 3 \mathrm{NH} 3 \mathrm{Pbl} 3$ perovskite films via adjustable volume expansion. Nano Lett. 15, 3959-3963 (2015).

20. $\mathrm{Fu}$, F. et al. Controlled growth of Pbl2 nanoplates for rapid preparation of $\mathrm{CH} 3 \mathrm{NH} 3 \mathrm{Pbl} 3$ in planar perovskite solar cells. Phys. Status Solidi A 212, 2708-2717 (2015) 
21. RefFITprogram. https://sites.google.com/site/reffitprogram/home.

22. Byrnes, S. J. Multilayer optical calculations. arXiv. http://arxiv.org/abs/ 1603.02720 (2016)

23. Morales-Masis, M., Nicolas, S. M. D., Holovsky, J., Wolf, S. D. \& Ballif, C. Lowtemperature high-mobility amorphous IZO for silicon heterojunction solar cells. IEEE J. Photovolt. 5, 1340-1347 (2015).

24. Pisoni, S. et al. Impact of interlayer application on band bending for improved electron extraction for efficient flexible perovskite mini-modules. Nano Energy 49, 300-307 (2018)

25. Bi, D. et al. Efficient luminescent solar cells based on tailored mixed-cation perovskites. Sci. Adv. 2, e1501170 (2016).

26. Xiao, Z. et al. Efficient, high yield perovskite photovoltaic devices grown by interdiffusion of solution-processed precursor stacking layers. Energy Environ. Sci. 7, 2619-2623 (2014).

27. Liu, D., K. Gangishetty, M. \& L. Kelly, T. Effect of CH 3 NH 3 Pbl 3 thickness on device efficiency in planar heterojunction perovskite solar cells. J. Mater. Chem. A 2, 19873-19881 (2014).

28. Liu, D. et al. Tailoring morphology and thickness of perovskite layer for flexible perovskite solar cells on plastics: the role of $\mathrm{CH} 3 \mathrm{NH} 3 \mathrm{I}$ concentration. Sol. Energy 147, 222-227 (2017)

29. Cao, D. H. et al. Remnant Pbl2, an unforeseen necessity in high-efficiency hybrid perovskite-based solar cells? APL Mater. 2, 091101 (2014).
30. Cheng, Y. et al. Spectroscopic study on the impact of methylammonium iodide loading time on the electronic properties in perovskite thin films. J. Mater. Chem. A 4, 561-567 (2016).

31. Stranks, S. D. et al. Electron-hole diffusion lengths exceeding 1 micrometer in an organometal trihalide perovskite absorber. Science 342, 341-344 (2013).

32. Poorkazem, K. Liu, D. \& L. Kelly, T. Fatigue resistance of a flexible, efficient, and metal oxide-free perovskite solar cell. J. Mater. Chem. A 3, 9241-9248 (2015).

33. Wang, Y. et al. High-efficiency flexible solar cells based on organometal halide perovskites. Adv. Mater. 28, 4532-4540 (2016)

34. Lee, H.-M., Kang, S.-B., Chung, K.-B. \& Kim, H.-K. Transparent and flexible amorphous In-Si-O films for flexible organic solar cells. Appl. Phys. Lett. 102 021914 (2013)

35. Morales-Masis, M., Wolf, S. D., Woods-Robinson, R., Ager, J. W. \& Ballif, C Transparent electrodes for efficient optoelectronics. Adv. Electron. Mater. 3 1600529 (2017)

36. Heo, G.-S., Gim, I.-G., Lee, H.-K., Song, J.-H. \& Kim, T.-W. Investigation of bending stability of amorphous $\mathrm{Zn}-\mathrm{In}-\mathrm{Sn}-\mathrm{O}$ thin films on flexible poly(ether sulfone) substrates. Jpn. J. Appl. Phys. 49, 031104 (2010).

37. Eerden, Mvan et al. Optical analysis of planar multicrystalline perovskite solar cells. Adv. Opt. Mater. 5, 1700151 (2017).

38. Armin, A. et al. Quantum efficiency of organic solar cells: electro-optical cavity considerations. ACS Photonics 1, 173-181 (2014). 